\title{
DESEMPENHO DE LÁPAROS LACTENTES SOB ACESSO LIVRE OU RESTRITO DA COELHA AO NINHO
}

\author{
Performance of Suckling Rabbits on Free or Restricted Access \\ of the Female to the Litter
}

\author{
Elaine Cristina Krygierowicz ${ }^{1}$ \\ Iris Patricia dos Santos ${ }^{1}$ \\ Patricia Aparecida Basniak ${ }^{1}$ \\ Rosana Adriane Skorei Ferreira ${ }^{1}$ \\ Marson Bruck Warpechowski
}

\section{Resumo}

Um experimento em granja comercial foi realizado para avaliar o efeito do acesso da coelha à ninhada de forma livre ou restrita uma vez ao dia até os 21 dias, sobre o desempenho dos láparos até a desmama, aos 30 dias. Foram avaliadas 84 ninhadas provenientes de 60 coelhas da raça Nova Zelândia Branco, distribuídas em delineamento, blocos ao acaso, com dois tratamentos: acesso livre ou controlado da fêmea ao ninho. O peso da ninhada foi obtido no nascimento e na desmama e os ninhos foram revisados duas vezes por semana. Houve interação significativa entre o manejo de acesso ao ninho e o número médio ponderado de láparos na ninhada $(\mathrm{P}=0,087)$. A análise de regressão entre o ganho de peso médio (GPM) em função número ponderado de láparos na ninhada (NPL) resultou nas equações: $\mathrm{GPM}=717,1-9,5^{*} \mathrm{NPL} ;(\mathrm{r}=-0,79 ; \mathrm{P}<0,001)$, para o acesso restrito, e GPM $=643,6-24,8 * \mathrm{NPL}(\mathrm{r}=-0,54 ; \mathrm{P}=0,002)$, para o acesso livre. Nas condições em que foi realizado o experimento, pode-se concluir que permitir apenas uma amamentação diária não é um manejo recomendável, uma vez que potencializou o efeito negativo do tamanho da ninhada sobre o ganho de peso dos láparos.

Palavras-chave: Amamentação; Coelhos; Desempenho; Ganho de peso; Manejo na lactação.

\section{Abstract}

A trial was conducted in a commercial rabbit farm to evaluate the effect of free access or once a day time restricted access of the female to the litter, just to 21 days, on performance of the rabbits just to the weaning, at 30 days of age. The 84 litters from 60 White New Zealand females were evaluated, in a randomized block design with two treatments: free or controlled access of the female to the litter. Litter weight was evaluated at birth and at weaning, and the litter was revised twice a week. There was significant interaction between the access management and the weighted mean litter size $(\mathrm{P}=0.087)$. The regression analyses of the mean weight gain $(\mathrm{GPM})$ as function of the weighted litter size (NPL) resulted in the equations: GPM $=717.1-$ 9.5* NPL; $(r=-0.79 ; \mathrm{P}<0.001)$, for the controlled access, and GPM $=643.6-24.8^{*} \mathrm{NPL}(\mathrm{r}=-0.54 ; \mathrm{P}=0.002)$, for the free access management. At the conditions in which the trial was conducted, it was concluded that to allow only a daily nursing is not a recommended management, while it provokes a negative effect of the litter size on the rabbits weight gain.

Keywords: Litter management; Nursing; Performance; Rabbits; Weight gain.

1 Alunas do Curso de Graduação em Zootecnia da Universidade Federal do Paraná, Curitiba, PR, Brasil.

2 Professor Dr. Adjunto do Departamento de Zootecnia, Setor de Ciências Agrárias, Universidade Federal do Paraná. Rua dos Funcionários, n. ${ }^{\circ}$ 1540, Curitiba, PR, Brasil. CEP 80035-050 (marson@ufpr.br) 


\section{Introdução}

O bom desempenho dos láparos lactentes depende, dentre outros fatores, do manejo das matrizes e da ninhada. Dentre os tipos de manejo de ninho citados por Vieira (1981), temos o livre acesso da coelha aos láparos com a utilização de ninhos abertos, inseridos na gaiola da fêmea anteriormente ao parto, e os ninhos instalados externamente à gaiola, munidos de portas que permitem restringir o acesso da coelha ao ninho conforme o manejo utilizado. A utilização de ninhos externos com a restrição de uma amamentação por dia é citada por diversos autores, com objetivos de pesquisa ou como manejo comercial associado à alta intensidade reprodutiva (VIEIRA, 1981; MCNITT; MOODY JR., 1988; MAERTENS; DE GROOTE, 1990; FARIA et al., 2004). Atualmente, essa prática é corrente em algumas produções cunículas da região metropolitana de Curitiba, PR, incluindo a granja onde foi realizado o presente trabalho. Entretanto, embora não tenha sido encontrado trabalho comparando esse manejo com o de acesso livre das fêmeas aos láparos, alguns pesquisadores têm sugerido que a restrição do número de amamentações possa resultar em prejuízo ao seu desempenho (MCNITT; MOODY JR., 1988; MAERTENS; DE GROOTE, 1990). Esse prejuízo é ainda maior quando se consideram ninhadas maiores (SCAPINELLO et al., 1999; FARIA et al., 2004).

O objetivo do presente trabalho foi avaliar o efeito do acesso da coelha à ninhada de forma livre ou restrita uma vez ao dia, sobre o desempenho dos láparos até a desmama.

\section{Materiais e métodos}

O estudo foi realizado em granja comercial localizada em Campo Largo-PR, no período de setembro de 2005 a fevereiro de 2006. Nesse experimento, foram avaliadas 84 ninhadas provenientes de 60 coelhas da raça Nova Zelândia Branco, primíparas e multíparas, distribuídas em dois tratamentos, acesso livre e controlado da coelha ao ninho, com 30 e 54 repetições (ninhadas), respectivamente.

Foram utilizados dois galpões. O primeiro galpão era construído em madeira, coberto com telhas de barro e com janelas munidas de cortinas reguláveis, possuindo gaiolas suspen- sas de arame galvanizado com 0,90 m de comprimento, $0,75 \mathrm{~m}$ de largura e $0,45 \mathrm{~m}$ de altura, contendo ninhos de madeira com $0,40 \mathrm{~m}$ de comprimento, 0,30 $\mathrm{m}$ de largura e $0,30 \mathrm{~m}$ de altura, em que normalmente se alojavam fêmeas com acesso livre à ninhada. O segundo galpão era construído em alvenaria, coberto com telhas de cimento amianto e com janelas munidas de cortinas reguláveis, contendo gaiolas suspensas do tipo espanhola, de arame galvanizado com 0,50 $\mathrm{m}$ de comprimento, 0,58 $\mathrm{m}$ de largura e 0,32 $\mathrm{m}$ de altura, munidos de ninhos de madeira presos externamente à gaiola, com $0,40 \mathrm{~m}$ de comprimento, 0,30 $\mathrm{m}$ de largura e 0,30 $\mathrm{m}$ de altura, em que normalmente se alojavam as fêmeas com acesso controlado à ninhada. Para avaliação de um possível efeito de galpão, cinco gaiolas de cada um deles foram adaptadas para efetuar o manejo contrário, ou seja: acesso controlado no primeiro galpão e livre no segundo. Para isso, cinco ninhos de colocação interna à gaiola foram adaptados com uma tampa para controlar o acesso da fêmea, e cinco ninhos externos foram mantidos permanentemente abertos para permitir acesso livre das fêmeas aos láparos. No manejo de acesso controlado, os ninhos foram abertos diariamente durante cinco minutos entre as 7 e as 8 horas da manhã. Após 21 dias de idade dos láparos, o acesso foi liberado permanentemente para entrada da fêmea e saída dos láparos, permanecendo assim até o desmame, realizado aos 30 dias de idade. Os láparos mortos foram retirados durante as revisões realizadas duas vezes por semana. O material utilizado como cama nos ninhos foi o capim seco ou maravalha, dependendo da disponibilidade. O efeito dos dois tipos de manejo foi avaliado pela mortalidade e ganho de peso dos láparos até o desmame. A pesagem das ninhadas foi realizada com balança eletrônica de precisão $0,05 \mathrm{~kg}$, no nascimento e no desmame. Foram calculados o peso médio ao nascer (PMN), ganho de peso médio dos láparos (GPM) e o número médio ponderado de láparos (NPL), obtido pela média de láparos vivos por dia até o desmame de cada ninhada, para correção do GPM.

O delineamento experimental foi o de blocos ao acaso, considerando os galpões como blocos, o manejo de acesso ao ninho como fator classificatório e o NPL e a ordem do parto como fatores quantitativos. As inclinações das retas re- 
sultantes dos modelos ajustados por meio de regressões lineares entre o NPL e as variáveis estudadas foram comparadas entre os manejos de acesso restrito e livre, para verificar a interação desses fatores. Para a análise da variável inicial, peso médio dos láparos ao nascer, foi utilizado delineamento completamente casualizado, considerando os efeitos de galpão, tipo de ninho e a ordem de parto das coelhas. As análises estatísticas foram realizadas utilizando o programa StatGraphics Plus for Windows 4.1 (MANUGISTICS, 1997).

\section{Resultados e discussão}

A ordem do parto não teve efeito significativo sobre nenhuma das variáveis estudadas $(\mathrm{P}>0,80)$, sendo por isso retirada do modelo. Não houve diferença significativa no peso médio ao nascer entre as ninhadas avaliadas para os dois tipos de manejo $(\mathrm{P}>0,71)$, não sendo, portanto, necessária a sua utilização como covariável na avaliação das variáveis de desempenho. Entretanto, conforme esperado, houve efeito significativo do número de láparos nascidos (NLN) sobre essa variável, que diminuiu com o aumento do tamanho da ninhada $(\mathrm{P}<0,01)$, conforme demonstrado no Gráfico 1.

\section{GRÁFICO 1 - Regressão linear entre o peso médio ao nascer (PMN) e o número de láparos na ninhada $(\mathrm{NL})$, a linha representa a resposta calculada $\left(\mathrm{PMN}=79,76-2,49^{*} \mathrm{NL} ; \mathrm{r}=-0,51 ; \mathrm{P}<0,001\right)$. Graph 1 -Linear regression between the weight to the rising average (PMN) and the number of suckling rabbits in the batch (NL), the line represents the calculated reply $\left(P M N=79.76-2.49{ }^{*} N L ; r=-0.51 ; P<0.001\right)$.}

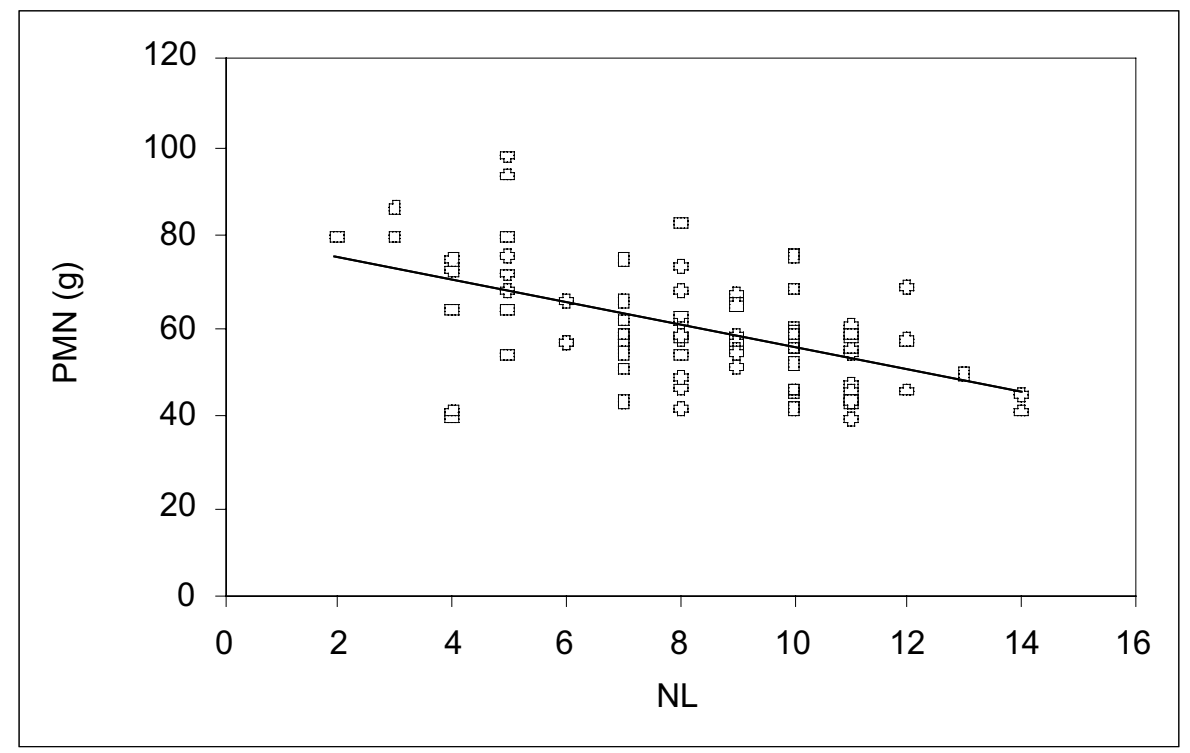

As médias de peso ao nascer (PMN), ganho de peso médio (GPM) e as médias de mortalidade para os dois tipos de manejo avaliado são apresentadas na Tabela 1 .

Não foi observado efeito significativo sobre a mortalidade, porém o alto coeficiente de variação observado $(72,5 \%)$ demonstra que os resultados obtidos não foram suficientes para avaliar estatisticamente o efeito dos fatores estudados sobre essa variável. O índice de mortalidade até a desmama, segundo Scandian (1999), é de
15\%. Entretanto, na propriedade em que foi realizado o presente estudo, a mortalidade atingiu um máximo de 20\%, segundo dados do proprietário, o que está de acordo com os dados obtidos no período experimental. McNitt e Moody Jr. (1988) também encontraram dificuldades na avaliação da mortalidade em ninhadas de láparos, o que pode ser atribuído ao grande impacto percentual que cada morte em ninhadas ocasiona, que normalmente não ultrapassam 14 animais. Apesar da impossibilidade de análise dos dados obtidos em seu 
trabalho quando comparavam um ou dois acessos das coelhas ao ninho, McNitt e Moody Jr. (1988) consideraram plausível aceitar que a maior disponibilidade de leite contribui para a maior sobrevivência de láparos lactentes. A diferença numérica na mortalidade observada entre os tratamentos do presente trabalho não contribui para essa afirma- ção. Entretanto, outros fatores devem ser considerados, como a provável melhor condição higiênica dos ninhos com acesso restrito da fêmea (dados não avaliados) e a observação diária no momento da mamada controlada, em comparação com a verificação padronizada duas vezes por semana realizada no restante da granja.

\section{TABELA 1 - Resultados de desempenho e mortalidade de láparos de ninhadas com acesso restrito ou livre da fêmea ao ninho'. \\ Table 1 - Performance and mortality of suckling rabbits of hatches with restricted access or exempt of the female to litter $^{1}$ results.}

\begin{tabular}{|c|c|c|c|}
\hline & $\operatorname{PMN}(g)^{2}$ & GPM $(g)^{3}$ & Mortalidade (\%) \\
\hline & & & Acesso da fêmea ao ninho \\
\hline Livre & 58,6 & 457,7 & 23,8 \\
\hline \multirow[t]{2}{*}{ Controlado } & 59,2 & 430,9 & 19,2 \\
\hline & & & Probabilidade dos efeitos \\
\hline Acesso ao ninho & 0,719 & 0,120 & 0,695 \\
\hline $\mathrm{NPL}^{4}$ & 0,001 & $<0,001$ & 0,209 \\
\hline Acesso x NPL & 0,305 & 0,087 & 0,957 \\
\hline$\overline{\mathrm{CV} \%}$ & 16,0 & 14,6 & 72,5 \\
\hline
\end{tabular}

Como pode ser observado na Tabela 1, houve interação significativa entre o manejo de acesso ao ninho e o número ponderado de láparos na ninhada sobre o ganho de peso dos láparos $(\mathrm{P}<0,09)$, sendo o efeito do NPL o mais importante $(\mathrm{P}<0,001)$. A relação entre o GPM e o NPL resultou em equações que demonstram o grande efeito negativo do tamanho da ninhada sobre o desempenho médio dos láparos (GRÁfICO 2). No manejo com acesso controlado da fêmea, o efeito foi maior, pois os láparos foram amamentados apenas uma vez ao dia, durante cinco minutos. Isso indica que quanto maior o número de láparos na ninhada, maior a chance de alguns deles não serem alimentados adequadamente na única oportunidade diária. Isso está de acordo com as afirmações de Vieira (1981), de que as coelhas amamentam as crias duas vezes por dia, e de Pinheiro Jr. (1973), que relata que as coelhas amamentam seus láparos várias vezes durante o dia e mesmo durante a noite. McNitt e Moody Jr. (1988) obser- varam ganho de peso até a desmama com 28 dias 80\% superior em ninhadas padronizadas de 8 láparos que recebiam duas amamentações diárias em comparação às que recebiam apenas uma. Esses autores observaram ainda que o efeito persistia após o desmame, com diferença próxima a 20\% aos 56 dias de idade dos láparos. Utilizando-se as equações apresentadas no Gráfico 1, o ganho de peso aos 30 dias padronizado para ninhadas de 8 láparos calculado com o manejo de acesso livre resultou em valor $11 \%$ superior ao calculado para o acesso restrito. A diferença aumentou para $42 \%$ ao considerar ninhadas de 12 láparos e diminuiu para - 3\% considerando ninhadas de 4 láparos. Faria et al. (2004), trabalhando com ninhadas de quatro e oito láparos, também observaram diminuição significativa no ganho de peso com o aumento no numero de láparos amamentados. Nesse trabalho, o ganho de peso foi $21 \%$ menor em ninhadas com oito láparos amamentados uma vez ao dia. Da mesma forma, Scapinello et al. (1999) 
haviam demonstrado ganho de peso maior em ninhadas de 4 láparos que de 10 láparos entre 16 e 32 dias de idade. Em ambos os trabalhos, o ganho de peso foi diretamente relacionado ao consumo médio de leite por láparo, que diminuiu com o aumento no tamanho da ninhada. Bianchini (2005) verificou que quanto mais cedo iniciar a ingestão de alimento sólido antes da desmama, menos problemas digestivos terão esses animais após a desmama. De acordo com Faria et al. (2004), a disponibilidade de leite para os láparos, modulada pelo número de filhotes da ninhada, número de mamadas por dia e produção de leite pelas fêmeas, interfere no início de consumo de alimentos sólidos e determina tanto o início da ingestão desses como o volume ingerido. Esses mesmos autores, referindo-se ao manejo de uma única amamentação por dia, consideram que isso impede que os láparos aproveitem todo o leite produzido pela fêmea, além de interferir no início do consumo de alimentos sólidos. Entretanto, Scapinello et al. (1999) haviam observado que o consumo de dieta de lactação pelos láparos iniciava apenas após os 18 dias, mesmo com restrição na amamentação, e que sua contribuição para o desempenho dos láparos era bem menor que a resultante do consumo de leite, além de não interferir na atividade enzimática intestinal e na capacidade de digestão de alimentos sólidos no pós-desmame. É possível inferir que a restrição para apenas um acesso da fêmea ao ninho mantém os láparos sem acesso a outra fonte de alimentação, o que pode prejudicar seu crescimento, especialmente com ninhadas maiores ou com fêmeas de menor produção de leite.

GRÁFICO 2 - Regressões lineares entre o ganho de peso médio da ninhada até o desmame (GPM) e o número ponderado de láparos na ninhada (NPL) de acordo com o manejo de acesso da coelha ao ninho. A linha pontilhada representa a resposta com acesso restrito $($ GPM $=717,1-39,5 * \mathrm{NPL}$; $r=-0,79 ; P<0,001) e$, a contínua, a resposta com acesso livre $\left(\mathrm{GPM}=643,6-24,8^{*} \mathrm{NPL} ; \mathrm{r}=-0,54\right.$; $\mathbf{P}=\mathbf{0 , 0 0 2}$ ).

Graph 2-Linear regressions between the hatch weight gain average until the wean (GPM) and the weighed number of suckling rabbits in the hatch (NPL) the handling of access of the rabbit to the nest. The dot line represents the reply with restricted access (GPM $\left.=717.1-39.5{ }^{*} N P L ; r=-0.79 ; P<0.001\right)$ and the continuum one, the reply with free access (GPM $\left.=643.6-24.8^{*} N P L ; r=-0.54 ; P=0.002\right)$.

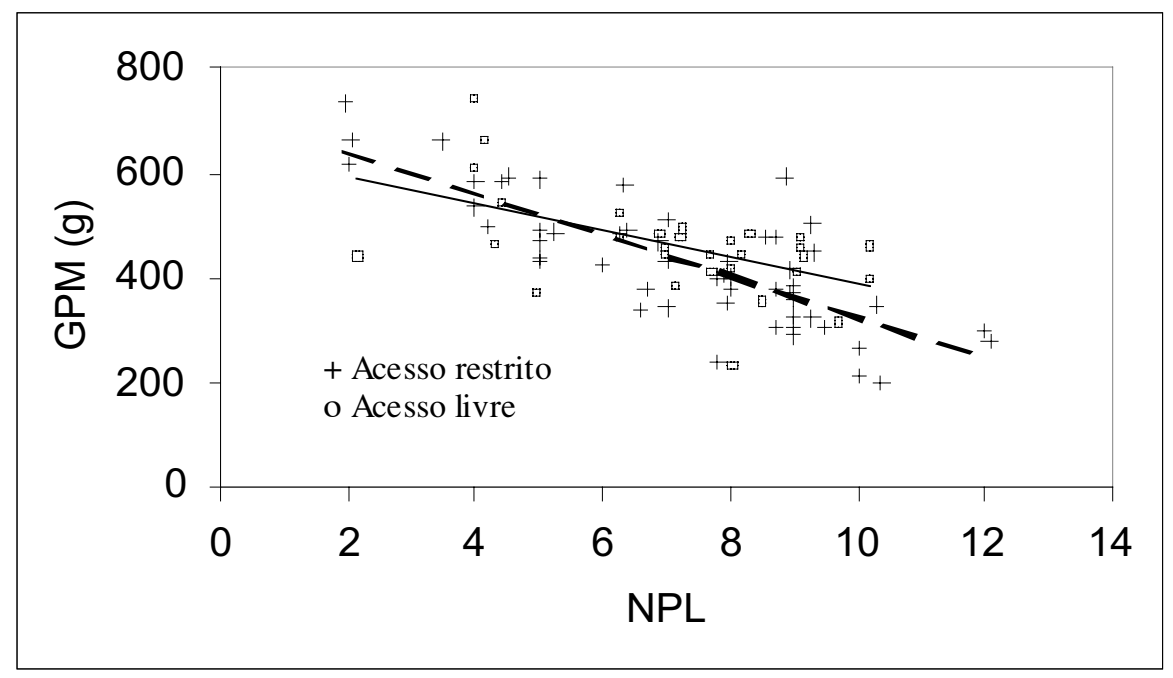

Por outro lado, outras possíveis vantagens de restrição do acesso da fêmea à ninhada, como melhor higiene do ninho, não foram diretamente avaliadas, mas os dados de desempenho e mortalidade não indicam melhorias nesse sentido. O efeito da permissão de mais de um acesso controlado ao ninho, bem como a avaliação de outras variáveis, como número de revisões semanais, temperatura interna do ninho, umidade da cama e variabilidade de peso da ninhada ao desmame poderiam ser importantes para melhor avaliação desse tipo de manejo. 


\section{Conclusões}

Nas condições em que foi realizado o experimento, pode-se concluir que permitir apenas uma amamentação diária potencializa o efeito negativo do tamanho da ninhada sobre o ganho de peso dos láparos. Baseado nisso, não se recomenda esse tipo de manejo, especialmente com grandes ninhadas.

\section{Agradecimentos}

Os autores agradecem ao Senhor Wanderley Marchetti e sua esposa Lígia Marchetti por ceder a propriedade e coelho para a execução do presente trabalho.

\section{Referências}

BIANCHINI, W. Influência do tamanho da ninhada e nível de amido nas dietas no desempenho de coelhos até a desmama. In: CONGRESSO NACIONAL DE ZOOTECNIA, 10, Campo Grande, 2005. Anais... Campo Grande: ABZ-UEMS, 2005. CD$\mathrm{ROM}$

FARIA, H. G., et al. Desempenho de coelhos até a desmama de acordo com o tamanho da ninhada e o nível de amido nas dietas. Rev. Bras. Zoot. Viçosa, v. 33, n. 4, p. 894-900, 2004.
MAERTENS, L.; DE GROOTE, G. Feed intake of rabbit kits before weaning and attempts to increase it. J. Appl. Rabbit Res. v. 13, p. 151-158, 1990.

MANUGISTICS. Statgraphics Plus for Windows. (versão 4.1). Rockville: Maryland, 1997.

McNITT, J. I.; MOODY JR., G. L. Milk intake and growth rates of suckling rabbits. J. Appl. Rabbit Res. v. 11, p. 117-119, 1988.

PINHEIRO JÚNIOR, G. C. Coelhos. Belo Horizonte: Itatiaia Limitada, 1973.

SCANDIAN, A. Coelho+técnica=lucro: alimentação, reprodução, doenças: profilaxia e tratamento. São Paulo: Nobel, 1999.

SCAPINELLO, C.; GIDENNE, T.; FORTUNLAMOTHE, L. Digestive capacity of the rabbit during the post-weaning period, acording to the milk/solid feed intake pattern before weaning. Reprod. Nutr. Dev. v. 39, p. 423-432, 1999.

VIEIRA, M. I. Coelhos: Instalações e acessórios. 7. ed. rev. e ampl. São Paulo, SP: o autor, 1981.

Recebido: 05/09/2005

Aprovado: 20/12/2005 